\title{
Recomendaciones para la prevención del cáncer dadas por el Fondo Mundial para la Investigación sobre Cáncer (FMIC): análisis de la situación en Chile
}

\author{
MIRTA CROVETTO ${ }^{1, \mathrm{a}}$, RICARDO UAUY ${ }^{2}$
}

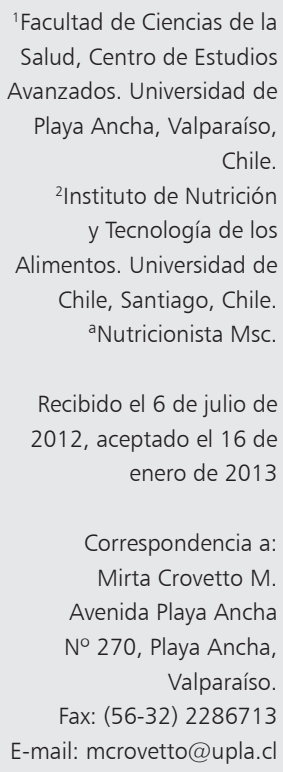

'Facultad de Ciencias de la Salud, Centro de Estudios Avanzados. Universidad de Playa Ancha, Valparaíso, Chile.

${ }^{2}$ Instituto de Nutrición y Tecnología de los Alimentos. Universidad de Chile, Santiago, Chile. ${ }^{a}$ Nutricionista Msc.

Recibido el 6 de julio de 2012, aceptado el 16 de enero de 2013

Correspondencia a: Mirta Crovetto M Avenida Playa Ancha № 270, Playa Ancha, Valparaíso.

Fax: (56-32) 2286713 E-mail: mcrovetto@upla.cl

\section{Recommendations for cancer prevention of World Cancer Research Fund (WCRF): situational analysis for Chile}

The main diet-related cancers include colorectal, lung, breast in (postmenopausal) women, stomach, esophagus, prostate and pancreas. After tobacco, obesity is the leading cause of cancer; it accounts for one third of all cancers. Cancer is associated with high total body fat, abdominal fat and weight gain in adult life. These are all potentially modifiable risk factors. Consumption of a "healthy diet" and living an "active life" can significantly reduce the risk of cancer. The aim of this study was to analyze the recommendations published by the World Cancer Research Fund (WCRF) and American Institute for Cancer Research (AICR) for the prevention of cancer in 2007. We compared the recommendations of "Food, Nutrition and Physical Activity and the Prevention of Cancer: a global perspective", with the national situation in Chile, analyzing the national report on the prevalence of risk factors. Our main finding was that the pattern of consumption and lifestyles differ markedly from the WCRF recommendations: we observed an over consumption of sugary drinks and high intake of processed foods high in sodium and total fat and low consumption of legumes, vegetables, fruits high in antioxidants and fiber that protect from cancer. Chile has an increased cancer prevalence which is associated with poor quality diets, rising mean body mass index and a sedentary behavior. We recommend the strengthening programs to promote healthy diets and active living, in order to reduce cancer risk.

(Rev Med Chile 2013; 141: 626-636).

Key words: Diet; Health Promotion; Neoplasms; Risk factors.
$\mathrm{E}$ 1 cáncer es la segunda causa de muerte en el mundo, según estadísticas de la Organización para la Cooperación y Desarrollo (OCDE), la tasa de mortalidad por todos los cánceres en el 2009 fue de 124 por 100 mil habitantes para mujeres y de 208 por 100 mil habitantes para hombres ${ }^{1}$. Según la evidencia científica, $40 \%$ de los cánceres se pueden prevenir mediante la reducción de los factores de riesgo y la prevención primaria, $30 \%$ adicional pueden ser curados con una detección temprana y tratamiento apropiado $\mathrm{y}$, todos los casos de cáncer avanzados pueden beneficiarse de los cuidados paliativos. Los cánceres se deben principalmente, a cinco factores de riesgo: dieta inadecuada, índice de masa corporal aumentada, falta de actividad física y, consumo de tabaco y alcohol. Asimismo, los causados por el virus de la hepatitis B (VHB) y C (VHC) o papiloma-virus humanos (PVH), que en los países de ingresos bajos y medios corresponden al 20\% de las muertes por cáncer². Más de 30\% de los cánceres se pueden prevenir con estilos de vida saludable ${ }^{2-6}$.

En Chile, en el 2009, el cáncer alcanzó al 25,6\% 
del total de defunciones por grandes grupos de muertes $^{7}$; en el 2010 se registraron en total 23.136 defunciones por cáncer, con una tasa de 135,3 por 100 mil habitantes ${ }^{8}$. Según sexo, los cánceres más frecuentes en las mujeres son el de mama, vesícula biliar y cuello uterino $(55,3 \%)$ y en los hombres el de próstata, estómago y piel $(58,7 \%)^{9-11}$.

En noviembre del año 2007, el Fondo Internacional para la Investigación del Cáncer (WCRF) y el Instituto Americano de Investigación del Cáncer (AICR) publicaron el informe "Alimentación, Nutrición, Actividad Física y la Prevención del Cáncer: una perspectiva mundial", elaborado por 21 expertos mundiales, a partir de la revisión y análisis de los resultados de 7.000 estudios científicos, que confirmaron la evidencia de la asociación entre distintos cánceres y la alimentación, la actividad física y el peso de las personas. El informe incluye un conjunto de diez recomendaciones que permiten disminuir las posibilidades de desarrollar el cáncer, que la AICR resumió en tres grandes directrices: "prefiera los alimentos vegetales, limite el consumo de carnes rojas y evite las carnes procesadas", "sea físicamente activo durante 30 min diarios como mínimo" y "mantenga un peso saludable durante toda la vida ${ }^{12}$.

En este contexto, el objetivo de este estudio fue analizar y comparar las recomendaciones propuestas por WCRF/AICR para evaluar los factores de riesgo en la población en Chile, orientar su prevención y servir para facilitar la toma de decisiones en políticas públicas con el objeto de disminuir la carga de enfermedad y muerte por cáncer en la población chilena.

\section{Material y Método}

El artículo se basa en los antecedentes del informe "Alimentación, Nutrición y Actividad Física y la Prevención del Cáncer: una perspectiva mundial" del WCRF/AICR del año 2007, para comparar las diez recomendaciones emanadas de este estudio con la situación nacional, en base a los estudios representativos de la situación de salud en Chile.

Se describen y analizan las 10 recomendaciones para resumir las relaciones entre el consumo de alimentos y el nivel de riesgo de presentar los diversos tipos de cáncer Tabla 1.

Las recomendaciones emanadas por el WCRF/
AICR son: 1) Mantener el peso dentro de los márgenes normales del peso corporal; 2) Mantenerse físicamente activo como parte de la vida cotidiana; 3) Limitar la ingesta de alimentos de alta densidad energética y evitar el consumo de bebidas azucaradas que promueven el aumento de peso; 4) Consumir sobre todo alimentos de origen vegetal; 5) Limitar la ingesta de carnes rojas y no consumir carnes procesadas; 6) Limitar el consumo de sal y no consumir cereales y legumbres contaminados por aflatoxinas; 7) Limitar el consumo de bebidas alcohólicas; 8) Lograr una nutrición adecuada sin recurrir a suplementos alimentarios. Recomendaciones especiales; 9) Lactancia natural. 10) Sobrevivientes de cáncer.

\section{Resultados}

\section{Mantención del peso corporal}

En Chile, la obesidad se ha incrementado desde 1960, año del primer estudio nacional del estado nutricional en mayores de 15 años que arrojó $15 \%$ y $17 \%$ de obesidad para hombres y mujeres, respectivamente ${ }^{13}$. En 1984/85, Bove (Bove MI. Prevalencia de obesidad en 4.241 adultos pertenecientes a diferente nivel socioeconómico de Santiago. Tesis Magíster PAN, INTA, Universidad de Chile. 1985) y Berríos en 1987/88, informan de $19,6 \%$ y $20 \%$ de obesidad en hombres y de $30,8 \%$ y $13 \%$ en mujeres, en el Gran Santiago, respectivamente ${ }^{14}$. En 1992, Berríos, en una sub muestra del grupo anterior informa de 39,3\% de obesidad en mujeres y de $20 \%$ en hombres, evidenciando que en menos de 5 años la obesidad se había duplicado ${ }^{15}$.

Las Encuestas Nacionales de Salud (ENSA) han confirmado esta tendencia. Las ENSA 2003 y 2010 indicaron una prevalencia de $22 \%$ y $25,1 \%$ en obesidad y de $38 \%$ y $39,3 \%$ de sobrepeso, respectivamente, más de 6 puntos porcentuales de aumento en el período; en el 2003, la obesidad central medida por la Circunferencia de Cintura (CC) fue de 90,7 cm en hombres y de $86,2 \mathrm{~cm}$ en mujeres (OMS, 1998) y en el $2010,62 \%$ a nivel nacional presentó obesidad central ${ }^{16,17}$.

El Sistema Nacional de Evaluación de Educación Física (SIMCE 2010) aplicada por primera vez a nivel nacional a estudiantes de $8^{\circ}$ básico, mostró que $40,2 \%$ tenía sobrepeso u obesidad y 9,2\% tenía una condición física satisfactoria ${ }^{18}$. 


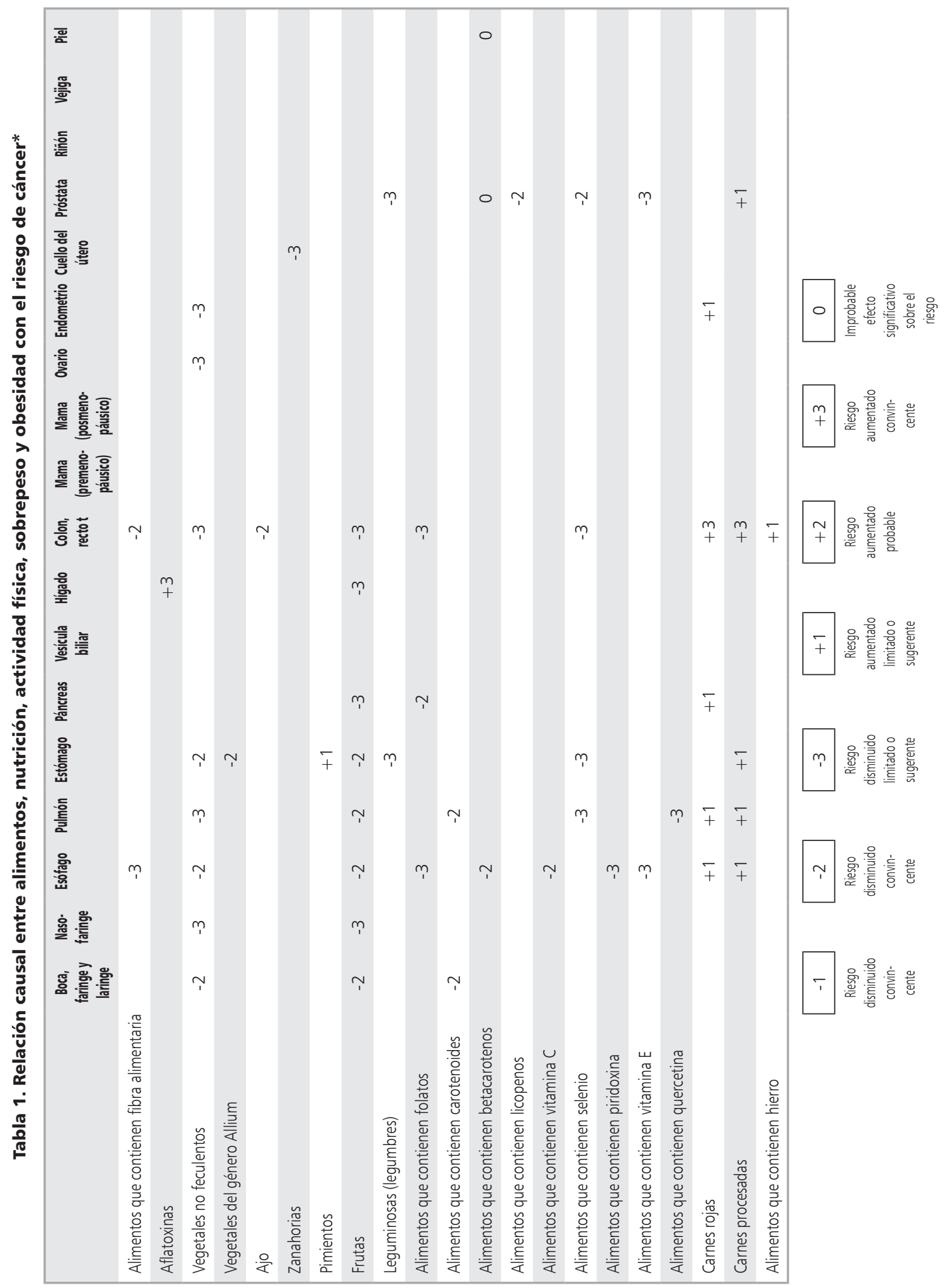


Recomendaciones para prevención de cáncer - M. Crovetto et al

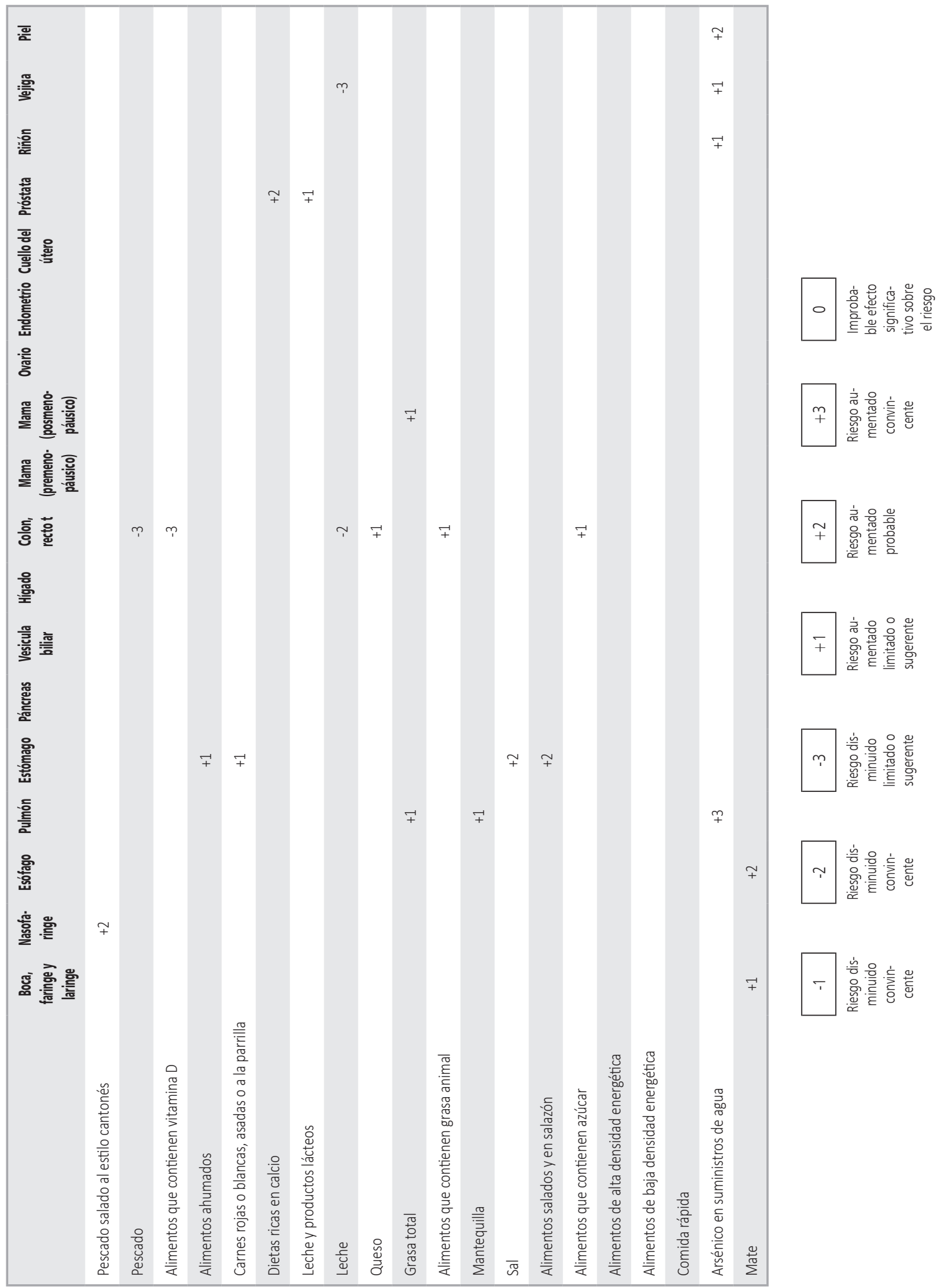




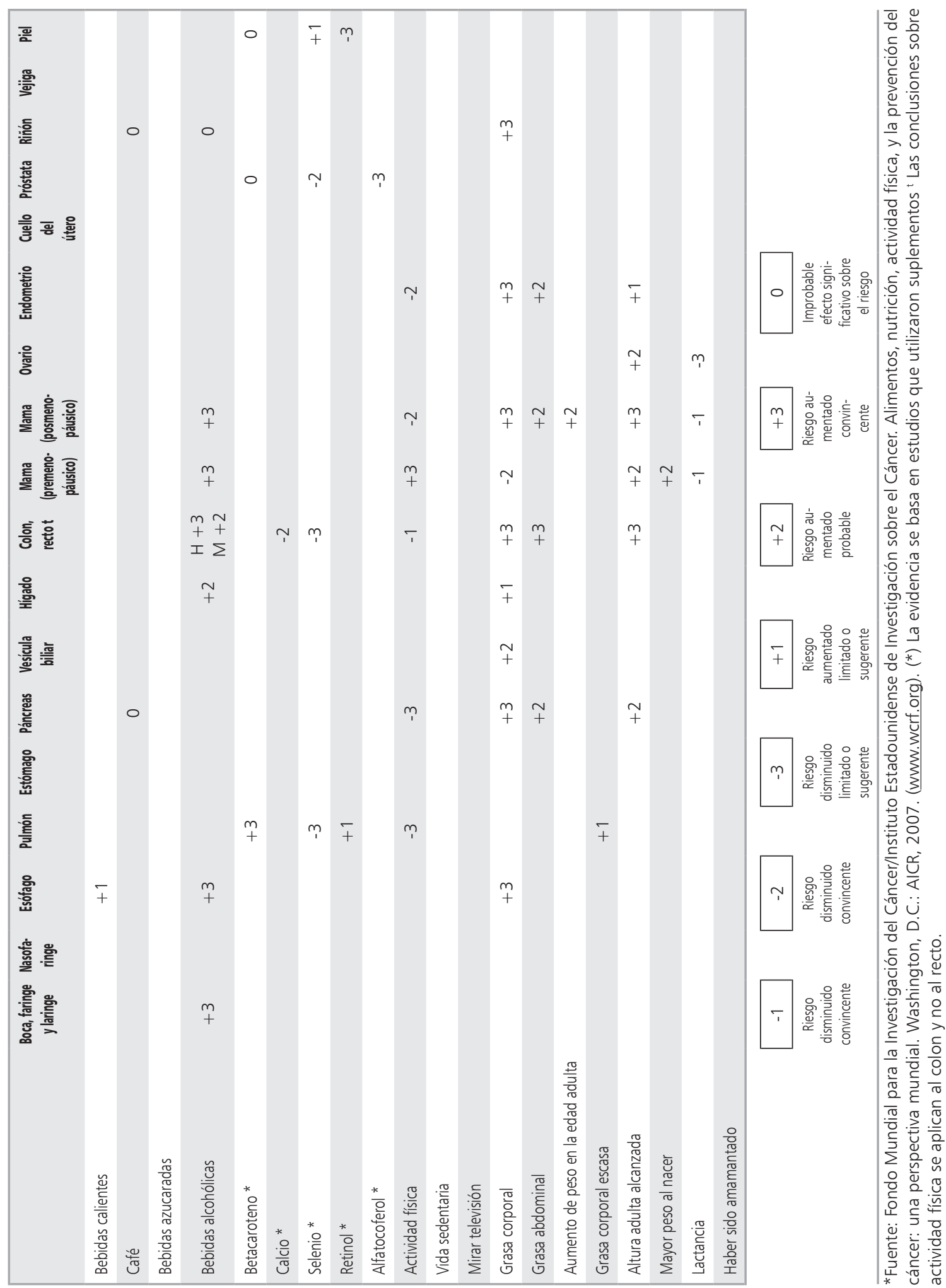


En la población infantil que ingresa a la educación básica, la obesidad varió de 7,6\% en 1987 a 21,5\% en el 2010 según datos de la Junta Nacional de Auxilio Escolar y Becas (JUNAEB) ${ }^{19}$.

\section{Mantención de la actividad física}

La población nacional es sedentaria, distintos estudios demuestran que la situación ha empeorado desde 1988; Berríos y col., en mayores de 15 años en la Región Metropolitana, encontró que $60 \%$ de los hombres y $80 \%$ de las mujeres realizaban menos de 15 min de actividad física continua, dos veces por semana ${ }^{14}$. Las ENSA 2003 y 2010, señalan que $89 \%$ de la población es sedentaria, sin mostrar diferencias entre ambas ${ }^{16,17}$.

El sedentarismo infantil se asocia a las h de permanencia frente al televisor, las que han ido aumentado desde 1995, observándose que 40,7\% de los niños estaba $3 \mathrm{~h}$ /día frente al televisor, más de $20 \%$ sobre tres horas/día y de 4 ó 5 h/día los fines de semana ${ }^{19,20}$.

\section{Alimentos y bebidas que promueven el aumento de peso}

En este ítem se incluyen los snacks dulces, salados y productos procesados que en general, se caracterizan por ser altos en densidad energética, grasas saturadas, azúcares agregados y sal. Se consideran alimentos de alta densidad energética a los que exceden de $250 \mathrm{kcal}$ por cada $100 \mathrm{~g}$. La densidad energética media debe disminuir a menos de $125 \mathrm{kcal}$, aproximadamente.

Chile tiene un consumo per cápita anual de 1,4 $\mathrm{kg}$ de snacks, $155 \mathrm{l}$ de bebidas gaseosas (más de un vaso diario), similar al de lácteos y 2,3 $\mathrm{kg}$ de dulces sin considerar chocolates ni galletas. Noventa por ciento de las bebidas gaseosas contienen azúcares agregados, en promedio una bebida de 350 cc tiene 3 a 4 cucharadas de azúcar con un aporte calórico de $140 \mathrm{kcal}^{22-24}$.

Estudios realizados por los autores señalan que entre 1987 y 2007, el consumo de azúcares agregados para el Total Hogar (TH) del Gran Santiago, varió de 68 g a 83 g/per cápita/día (18 a 22 cucharaditas/día) de los cuales, 3 y 13 cucharaditas se consumen en bebidas y jugos ${ }^{25,26}$.

En relación a la ingesta de snacks y bebidas gaseosas en escolares, estudios realizados en la Región Metropolitana a inicios de 2000, diferenciados por nivel socioeconómico (NSE), mostraron que en escolares entre 10 y 13 años, el consumo de snacks era de $78 \mathrm{~g} /$ día y $85 \mathrm{~g} /$ día en los de menor NSE versus 44 g/día y 51 g/día en los de NSE alto; en bebidas azucaradas, las niñas de NSE bajo consumían $229 \mathrm{ml}$ versus $171 \mathrm{ml} /$ día en las de NSE alto ${ }^{19}$. Estudios posteriores informaron de un consumo entre $343 \mathrm{~g}$ a $460 \mathrm{~g}$ diarios en escolares de tres regiones del país ${ }^{20,27}$.

\section{Consumo de alimentos de origen vegetal}

Se recomienda un consumo medio de hortalizas pobres en almidones y, frutas de al menos 600 $\mathrm{g} /$ día. Los cereales con poco o sin procesamiento (granos integrales) y las leguminosas (legumbres), así como otros alimentos que son fuente natural de fibras dietarias, deben aportar un promedio/per cápita/día no menor de $25 \mathrm{~g}$ /día de carbohidratos complejos no amiláceos.

En Chile, el consumo de frutas y hortalizas en los adultos es menor a lo recomendado. Las encuestas de Calidad de Vida 2000 y 2006, señalaron que $42 \%$ de los hombres y $52 \%$ de las mujeres consumían fruta todos los días, cifra que se mantuvo en el 2006 con un consumo de 166 g/per cápita/ día ${ }^{16,29}$. La ENSA 2010, señaló que $15,7 \%$ de la población consume 5 o más porciones de fruta y verduras y $13,8 \%$ cereales integrales ${ }^{17}$.

Estudios realizados por los autores entre 1987-2007, señalan un consumo percápita/día de hortalizas, legumbres y frutas para el TH del Gran Santiago de 240 g y 390 g, respectivamente ${ }^{30-32}$ (Figura 1).

\section{Limitar consumo de alimentos de origen animal}

El consumo de carnes rojas no debe exceder los 300 g por semana, con una mínima proporción (o ninguna) de carnes procesadas.

En Chile, el Instituto Nacional de Estadísticas (INE), señaló que en el año 2011, el consumo per cápita/año de carnes rojas fue de 38,2 k (18,7 k de vacuno y de $19,5 \mathrm{k}$ de cerdo $)^{33}$.

Las tres últimas Encuestas de Presupuestos Familiares (EPF) indican que para el TH del Gran Santiago, el consumo de carnes rojas y procesadas ha variado de $90 \mathrm{~g}$ (IV EPF) a $110 \mathrm{~g}$ (VEPF) y $80 \mathrm{~g}$ (VIEPF) per cápita/día, con un consumo semanal per cápita de 560 g, 730 g y 630 g, del cual 90 g, 320 g y 150 g corresponden a carnes procesadas, respectivamente $^{30-32}$ (Figura 2).

Existe una asociación positiva entre el elevado consumo de carnes rojas y en especial las procesadas y el cáncer colorrectal. También, es importante 

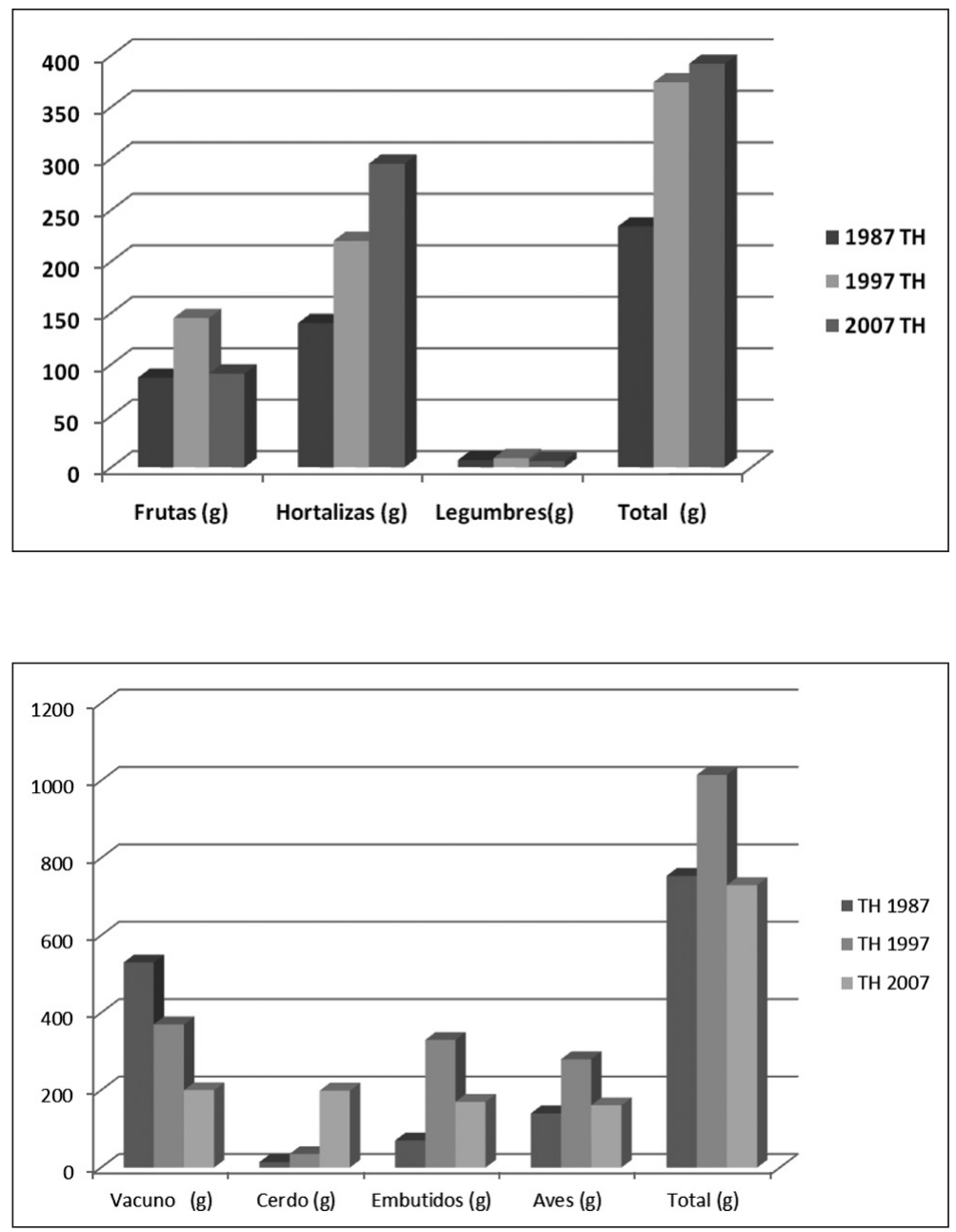

Figura 1. Consumo aparente. Frutas. Hortalizas. Legumbres. Gran Santiago total hogares 1987-1997-2007 (percápita/día). Fuente: Elaboración propia en base a IV, $\vee$ y VI EPF. INE.
Figura 2. Consumo aparente carnes. Total hogares. Gran Santiago. 19871997-2007 (percápita/semana). Fuente: Elaboración propia. En base a IV, V y VI Encuestas de Presupuestos Familiares. INE. señalar que se encontró una relación negativa entre esta enfermedad y el consumo de pescado ${ }^{34,35}$. Otros estudios han determinado que un bajo consumo de carnes rojas y procesadas, así como un mayor consumo de aves de corral y pescado podría reducir el riesgo de cáncer de ovario ${ }^{36,37}$.

\subsection{Limitar el consumo de sal}

El consumo medio de la población de sal (cloruro de sodio) procedente de cualquier fuente debe ser inferior a $5 \mathrm{~g}$ /día ( $2 \mathrm{~g}$ de sodio).

La ENSA 2010, mostró que los mayores de 15 años consumen en promedio 9,8 g de sal al día. Una proporción importante de esta ingesta de sal está relacionada con el consumo de productos elaborados y procesados ${ }^{17}$.
Según datos de la OMS, el cáncer de estómago a nivel mundial es aproximadamente de 980.000 casos de los cuales $4 \%$ podría evitarse limitando el consumo de sal ${ }^{38}$.

\subsection{No Consumir cereales y legumbres con aflatoxinas}

La exposición a las aflatoxinas de cereales y legumbres contaminadas por hongos debe reducirse al mínimo.

La contaminación con aflatatoxinas se origina por el crecimiento del hongo en productos como granos y cereales, frutos secos, frutas deshidratadas, leche y productos lácteos, hierbas, especias, café, cacao, aceites vegetales, cerveza, entre otros; en condiciones ambientales de contaminación 
Recomendaciones para prevención de cáncer - M. Crovetto et al

con humedad, agua disponible y elevadas temperaturas $^{39,40}$.

Según la OMS, las aflatoxinas tienen efectos tóxicos inmediatos son inmunosupresores, mutagénicos y carcinogénicos, afectando principalmente al hígado ${ }^{41}$.

\section{Limitar consumo de bebidas alcohólicas}

Una unidad de alcohol contiene entre $10 \mathrm{~g}$ a $15 \mathrm{~g}$ de etanol. El consumo de bebidas alcohólicas no debe exceder de dos unidades diarias en los varones y una en las mujeres. En Chile, el Primer Estudio Nacional de Drogas (1994) y la IX Encuesta Nacional de Drogas (2010) en la población entre 12-64 años señalan que la prevalencia de consumo de alcohol ha aumentado de $40 \%$ a $46,6 \%$, respectivamente ${ }^{42,43}$.

La ENSA 2010, mostró que el consumo semanal promedio de alcohol puro en Chile es de 88,4 g y la media de alcohol puro/g/día es superior a los $55,6 \mathrm{~g}$, este no debería ser mayor a los $20 \mathrm{~g}$ diarios. Estos datos son semejantes con el estudio de Carga de Enfermedad y Carga Atribuible al Alcohol en Chile (MINSAL 2007) ${ }^{17,10}$.

\section{Suplementos alimentarios}

Aumentar la proporción de la población que alcance una nutrición adecuada sin recurrir a suplementos alimentarios.

El comité de expertos considera que la mejor fuente de una buena nutrición son los alimentos y las bebidas naturales. En Chile, al igual que la situación mundial, existe un aumento notable en el consumo de suplementos nutricionales en la forma de pastillas, bebidas y alimentos especiales; no existe evidencia que apoye el consumo de suplementos nutricionales en la prevención del cáncer ${ }^{44}$.

\section{Recomendaciones especiales}

\section{Lactancia Materna}

La lactancia protege tanto a la madre como al niño. La lactancia materna exclusiva en Chile de 3 a 6 meses de duración entre 1993 y el 2005 ha aumentado de $46,4 \%$ y de $16 \%$ a $59,6 \%$ y $45,8 \%$, respectivamente ${ }^{45}$.

La lactancia materna por sobre los tres meses disminuye el riesgo e incidencia del cáncer de mama, especialmente en mujeres con antecedentes familiares y personales de riesgo de cáncer ${ }^{46,47}$.

\section{Sobrevivientes de cáncer}

Los sobrevivientes de cáncer viven permanentemente con el temor a la recurrencia del cáncer. No se sabe claramente la razón por la que el cáncer recurre en algunas personas y no en otras; realizar cambios de hábitos y conductas puede que no evite que el cáncer recurra, no obstante, puede mejorar la calidad de vida de las personas y evitar otros problemas de salud ${ }^{48,49}$. Se debe procurar seguir las recomendaciones de alimentación saludable, lograr un peso saludable y actividad física regular adecuada a sus requerimientos; a menos que hayan recibido otras indicaciones por parte de un profesional de la salud ${ }^{49,50}$.

\section{Conclusiones finales}

Los antecedentes presentados en este artículo señalan que en Chile a nivel poblacional existe un perfil de riesgo de cáncer elevado, asociado a las conductas alimentarias, actividad física, consumo de tabaco y alcohol y, altos índices de obesidad incompatibles con la salud. El patrón de consumo de alimentos y los indicadores analizados se alejan de las recomendaciones dadas por la OMS y la FAO, estando excedidos en los consumos recomendados de los alimentos que aumentan el riesgo y por debajo en los alimentos que tienen un efecto protector ${ }^{3,51,52}$.

Existe suficiente información científica que documenta el rol de la obesidad infantil, como un determinante importante de la obesidad en la vida adulta, factor de riesgo significativo para el desarrollo de las enfermedades crónicas no transmisibles (ECNT), las cardiovasculares y algunos tipos de cáncer ${ }^{51,52}$.

Hay diversas evidencias que señalan el rol protector de la actividad física en la mantención y rehabilitación de la salud, en especial las cardiovasculares y las ECNT. Las recomendaciones indican que el tiempo destinado a las actividades moderadas e intensas debería ser de al menos 60 min diarios en los niños ${ }^{49}$ y en los adultos de al menos 150 min de intensidad moderada o $75 \mathrm{~min}$ de actividad intensa ${ }^{52}$.

La Asociación Estadounidense del Corazón (AHA) recomienda limitar la cantidad de azúcares agregados en la alimentación. Las mujeres no deben obtener más de 100 calorías por día, provenientes del azúcar (aproximadamente, 6 
cucharaditas de azúcar) y los hombres no deben obtener más de 150 calorías por día provenientes del azúcar (aproximadamente 9 cucharaditas de azúcar) $)^{53,54}$.

El consumo de alimentos vegetales aportadores de fibra dietaria y fuente de antioxidantes, según datos de los autores el TH del Gran Santiago (VIEPF) se aproxima al mínimo de consumo de $400 \mathrm{~g} /$ día recomendado por la OMS los que aportan $12 \mathrm{~g} /$ per cápita/día de fibra dietaria, que es menos de la mitad de los $25 \mathrm{~g} /$ per cápita/día recomendado por los organismos internacionales ${ }^{51}$.

Los datos de la ENSA 2010 sobre el consumo de etanol ( $\mathrm{g} /$ día) se alejan de todas las recomendaciones internacionales con inicio de alto consumo a temprana edad ${ }^{17,55,56}$.

En la actualidad, la dieta de la población chilena se encuentra conformada por: 1) productos ricos en azúcares simples de alta carga glicémica, como las bebidas gaseosas y jugos endulzados con azúcar; 2) productos bajos en fibra dietaria (cereales integrales, legumbres, hortalizas y frutas); 3 ) fuentes proteicas en base a carnes y productos procesados de alto contenido en sodio, aditivos, grasas saturadas y densidad energética que junto con las prácticas culinarias de asar y ahumar presentan un riesgo para la salud respecto a carnes blancas, pescados y productos de origen vegetal; 4) productos procesados como snacks, galletas, pastelería, comidas preparadas, entre otros.

En conclusión, las recomendaciones emanadas del informe de WCRF/AICR tienen vigencia en nuestra sociedad ya que si los factores de riesgo persisten en el tiempo es esperable un aumento progresivo en la incidencia de cáncer tanto en nuestro país como a nivel internacional. Se estima que a nivel mundial la incidencia de cáncer al año 2030 alcanzará la cifra de 13,1 millones de habitantes, aumentando en $75 \%{ }^{57,58}$.

Por esto, es importante que los hábitos alimentarios cambien; una alimentación saludable debe incorporar alimentos protectores, con el objeto de que contribuyan a la recuperación y mantención de la salud y debe ser acorde con las orientaciones presentes en las guías alimentarias para la población chilena. Asímismo debe existir un cambio en las conductas poco saludables (peso corporal elevado por sobre lo normal, sedentarismo, consumo de alcohol y sal entre otros), con el fin de disminuir los factores de riesgo que aumentan la probabilidad de cáncer en la población general.

\section{Recomendaciones}

Se precisa una mayor acción del Estado y de los profesionales de la salud en educación alimentaria y estilos de vida saludable, focalizando en los preescolares, -los adultos del futuro- los que tendrán que aprender hábitos y conductas saludables desde su infancia. Así como regular, legislar y fiscalizar sobre los factores que afectan la salud, midiendo el impacto de las acciones efectuadas para asegurar que sean efectivas y eficaces.

La baja conciencia social de la relación de la dieta con las ECNT, el desarrollo silencioso de los factores de riesgo, la mayoría modificable con cambios conductuales y detección temprana, impide que se actúe oportunamente.

\section{Referencias}

1. OECD Health Data 2011. Health at a Glance 2011: OECD Indicators. All cancers mortality rates, males and females, 2009 (or nearest year). Disponible en: http:// dx.doi.org/10.1787/888932523424.

2. Organización Mundial de la Salud (OMS). GlOBOCAN 2008. (IARC). Sección de Información sobre el Cáncer (07/11/2012).

3. Organización Mundial de la Salud (OMS). Prevención y control de las enfermedades no transmisibles: aplicación de la estrategia mundial. Informe de Secretaría 61 ${ }^{\mathrm{a}}$ Asamblea Mundial de La Salud. Disponible en: http:// apps.who.int/gb/ebwha/pdf_files/A61/A61_8-sp.pdf.

4. Organización Mundial de la Salud (OMS). Informe sobre la salud en el mundo 2002 - Reducir los riesgos y promover una vida sana. Ginebra, Suiza, 2002.

5. Organización Panamericana de Salud. Organización Mundial de la Salud OPS/OMS: Estrategia Regional y Plan Integrado sobre el control y prevención de las Enfermedades Crónicas. Washington, D.C: OPS, USA 2007.

6. González CA, Riboli E. Diet and cancer prevention: Contributions from the European Prospective Investigation into Cancer and Nutrition (EPIC) study. Eur J Cancer 2010; 46 (14): 2555-62.

7. Departamento de Estadísticas e Información en Salud, Ministerio de Salud. Defunciones por grandes grupos de causas. Chile 2009. Disponible en: http://deis.minsal. cl/deis/Grafico_Morta_70_04\%20versi\%C3\%B3n\%20 4\%20(2)_archivos/Graficos_Grandes_causas_por\%20 a\%C3\%B1os_1960_2009.htm

8. Departamento de Estadísticas e Información en Salud, Ministerio de Salud. Distribución porcentual de las defunciones por grandes grupos de causas de muerte. 
Chile 1960, 1970, 1980, 1990, 2000, 2009. Disponible en: http://deis.minsal.cl/deis/Grafico_Morta_70_04\%20 versi\%C3\%B3n\%204\%20(2)_archivos/Defunciones_ Grandes_causas_tabla\%20y\%20graficos.htm

9. Departamento de Estadísticas e Información en Salud, Ministerio de Salud. Mortalidad por causas, según sexo. Chile 2000-2010. Disponible en: http://deis.minsal. cl/vitales/vitales2010/Serie_Mortalidad_causa_Chile_2000_2010/Chile.htm

10. Ministerio de Salud. Informe final: Estudio de carga de enfermedad y carga atribuible. Santiago, Chile: MINSAL. 2008.

11. Ministerio de Salud. Defunciones grandes causas. Chile. http//deis-minsal.cl

12. World Cancer Research Fund, American Institute for Cancer Research, Food, Nutrition and Physical Activity and the Prevention of Cancer: A Global Perspective Washington DC, USA. 2007.

13. Interdepartmental Committee on Nutrition for National Defense.Chile: Nutrition Survey, March-June 1960, National Institutes of Health, Bethesda, Maryland, USA, 1961.

14. Berríos X, Jadue L, Zenteno J, Ross MI, Rodríguez H. Prevalencia de factores de riesgo de enfermedades crónicas: un estudio poblacional en la Región Metropolitana de Santiago, Chile, 1986-1987. Rev Med Chile 1990; 118: 597-604.

15. Berríos X. Factores de riesgo en enfermedades crónicas de adultos. Un ejemplo de investigación epidemiológica. Bol Esc Med Universidad Católica de Chile 1994; 23: 73-89.

16. Ministerio de Salud (MINSAL). Encuesta Nacional de Salud: Informe final. Santiago: MINSAL, 2003.

17. Ministerio de Salud (MINSAL). Encuesta Nacional de Salud: Informe preliminar. Santiago: MINSAL, 20092010.

18. Ministerio de Educación. Informe de resultados Educación Física SIMCE 2010- $8^{\circ}$ Educación Básica. Disponible en: http://www.cooperativa.cl/prontus_nots/ site/artic/20110309/asocfile/20110309122818/informe_de_resultados_educacion_fisica_1.pdf

19. Junta Nacional de Auxilio Escolar y Becas. Situación nutricional de los escolares chilenos de $1^{\circ}$ Básico. Santiago, Chile: JUNAEB. Disponible en: http://bpt.junaeb. cl:8080/MapaNutricionalGx/.Consultado 24 de marzo, 2012.

20. Olivares S, Albala C, García F, Jofré I. Publicidad televisiva y preferencias alimentarias en escolares de la Región Metropolitana. Rev Med Chile 1999; 127: 791-9.

21. Olivares S, Kain J, Lera L, Pizarro F, Vio F, Moron C. Nutritional status, food consumption and physical acti- vity among Chilean school children: a descriptive study. Eur J Clin Nutr 2004; 58: 1278-85.

22. Latin American Market. Consumo de snacks en Chile. Disponible en: http://www.estrategia.cl/histo/200601/09/ ambito/snac.htm

23. Chilealimentos. Asociación de empresas de alimentos, Chile. Caramelos, chocolates y otros dulces. Diario Estrategia. Mayo, 2011. Disponible en: http://www.chilealimentos.com/link.cgi/Servicios/noticiero/estudio_mercado_coyuntura_2011/Caramelos_chocolates_otros $\% 20$ alimentos/18083.

24. Chilealimentos. Asociación de empresas de alimentos, Chile. Caramelos, chocolates y otros dulces. Diario Las Últimas Noticias, enero, 2012. Disponible en: http:// www.chilealimentos.com/link.cgi/Servicios/noticiero/2012_ESTUDIO_mercado_coyuntura/Caramelos_chocolates_otros/20538.

25. Crovetto M, Uauy R. Cambios en el consumo aparente de nutrientes en el Gran Santiago 1988-1997 en hogares según ingreso y su probable relación con patrón de enfermedades crónicas no trasmisibles. Rev Med Chile 2010; 138: 1091-108.

26. Crovetto M, Uauy R. Evolución del gasto en alimentos procesados en la población del Gran Santiago en los últimos 20 años Rev Med Chile 2012; 140: (3) (2012).

27. Lera L, Olivares S, Leyton B, et al. Patrones alimentarios y su relación con sobrepeso y obesidad en niñas chilenas de nivel socioeconómico medio alto. ALAN 2006; 56: 165-70.

28. Ministerio de Salud (MINSAL). I Encuesta Nacional de Calidad de Vida y Salud. Santiago: MINSAL, 2000.

29. Ministerio de Salud. II Encuesta de Calidad de Vida y Salud. Santiago: Departamento de Epidemiología y Departamento de Promoción de Salud, MINSAL, 2006.

30. Instituto Nacional de Estadísticas. IV Encuesta de presupuestos Familiares 1987-1988. Estructura del gasto de los hogares del Gran Santiago por grupo quintil de hogares. Santiago, Chile: INE. 1989.

31. Instituto Nacional de Estadísticas. V Encuesta de presupuestos familiares 1996-1997. v.3 Estructura del gasto de los hogares del Gran Santiago por grupo quintil de hogares. Santiago, Chile: INE. 1999.

32. Instituto Nacional de Estadísticas. VI Encuesta de presupuestos familiares 2006-2007. v.3 Estructura del gasto de los hogares del Gran Santiago por grupo quintil de hogares. Santiago, Chile: INE. 1999.

33. GC Retail in Detail. Consumo Carnes. Disponible en http://www.gcretailindetail.com/noticias-centrosudamerica/Chile/2012/01/10/Consumo-per-capita-decarne-en-Chile-es-de-842-Kg-al-ano/

34. Norat T, Bingham S, Ferrari P, Slimani N, Jenab M, 
Mazuir M, et al. Meat, fish, and colorectal cancer risk: the European Prospective Investigation into cancer and nutrition. J Natl Cancer Inst 2005; 97 (12): 906-16.

35. Morrison DS, Batty GD, Kivimaki M, Davey Smith G, Marmot M, Shipley M. Factores de riesgo de colon y rectal cáncer mortalidad: la evidencia de 40 años de seguimiento en el estudio Whitehall I. J Epidemiol Community Health 2011; 65 (11): 1053-8. Epub 2011 Mar 9.

36. Kolahdooz F, van der Pols JC, Bain CJ, Marks GC, Hughes MC, Whiteman DC, et al. Meat, fish, and ovariancancerrisk: Resultsfrom 2 Australian case-control studies, a systematic review, and meta-analysis. Am J Clin Nutr 2010; 91 (6): 1752-63. Epub 2010 Abr 14.

37. Kolahdooz F, Ibiebele TI, van der Pols JC, Webb PM. Dietary patterns and ovarian cancer risk. Am J Clin Nutr 2009; 89 (1): 297-304. Epub 2008 Dec 3.

38. RT, Actualidad/ciencia. Reducir el consumo de sal disminuye el riesgo de cáncer de estómago. RT http:// actualidad.rt.com/ciencias/view/49868-Reducir-consumo-de-sal-disminuye-riesgo-de-c\%C3\%A1ncer-deest $\% \mathrm{C} 3 \% \mathrm{~B} 3$ mago.

39. Zamora-Barquero Henry. Aflatoxinas B1 y su asociación con el cáncer de hígado. Acta Med Costarricense [revista en la Internet]. 2004 oct [citado 2012 oct 30]; 46 (4): 173-173. Disponible en: http://www.scielo.sa.cr/ scielo.php?script $=$ sci_arttext\&pid $=$ S0001-6002200 4000400003\&lng=es.

40. Bolet Astoviza M, Socarrás M. Micotoxinas y cáncer. Rev Cubana Invest Biomed [revista en la Internet]. 2005 Mar [citado 2012 oct 30]; 24 (1): 54-59. Disponible en: http://scielo.sld.cu/scielo.php?script=sci_arttext \&pid $=$ S0864-03002005000100007\&lng=es.

41. Chile Potencia Alimentaria. Aflatoxinas en los alimentos. http://www.chilepotenciaalimentaria.cl/content/ view/3127/Aflatoxinas-en-alimentos.html

42. Consejo Nacional para el Control de Estupefacientes (CONACE) Estudio Nacional de Drogas en Población General de Chile, 1994. CONACE Chile, 1996.

43. Consejo Nacional para el Control de Estupefacientes (CONACE) IX Estudio Nacional de Drogas en la Población General de Chile. 2010. CONACE, Chile, 2011.

44. González C. Nutrition and cancer: the current epidemiological evidence. BBr J Nutr 2006; 96 Suppl 1: S42-5.

45. Ministerio de Salud. Manual de Lactancia Materna 2010. Chile 2011.

46. Camayo J. Lactancia materna y cáncer de mama: un estudio caso-control en pacientes del Hospital Nacional Arzobispo Loayza, Lima-Perú. An Facmed. [revista en la Internet]. 2008 Mar [citado 2012 oct 30]; 69 (1): 22-8. Disponible en: http://www.scielo.org.pe/pdf/afm/v69n1/ a05v69n1.pdf
47. Aguilar M, González E, Álvarez J, Padilla C, Mur N, García P, Valenza M. Lactancia materna: un método eficaz en la prevención del cáncer de mama. Nutr Hosp. [revista en la Internet]. 2010 mar [citado 2012 oct 30]; 25 (6): 954-958. Disponible en: http://www.nutricionhospitalaria.com/pdf/4994.pdf

48. Instituto Nacional del Cáncer. La atención médica después del tratamiento del cáncer. Siga adelante: la vida después del tratamiento del cáncer. http://www.cancer. gov/espanol/cancer/siga-adelante/page3\#C5.

49. Rock C, Doyle C, Demark-Wahnefried W, Meyerhardt J, Courneya K, Schwartz A, et al. Nutrition and physical activity guidelines for cancer survivors. CA Cancer. J Clin 2012; 62 (4): 243-74. doi: 10.3322/caac.21142. Epub 2012 Apr 26.

50. Brown J, Byers T, Doyle C, Coumeya K, Demark-Wahnefried W, Kushi L, McTieman A, et al. The American Cancer Society guide for nutrition and physical activity for cancer survivors: a call to action for clinical investigators. CA Cancer. J Clin 2003; 53 (5): 268-91.

51. Organización Mundial de la Salud (OMS). Estrategia Mundial sobre régimen alimentario, actividad física y salud. En: 57a Asamblea Mundial de la Salud. Organización Mundial de la Salud. Ginebra, Suiza; OMS, 2004.

52. Organización Mundial de la Salud (OMS), Organización de las Naciones Unidas para la Agricultura y la Alimentación (FAO). Dieta, nutrición y prevención de enfermedades crónicas. En: Informe de una Consulta Mixta de Expertos. Serie de Informes Técnicos, 916. Ginebra, Suiza, 2003.

53. U.S. Department of Agriculture and U.S. Department of Health and Human Services. Dietary Guidelines for Americans, 2010. 7th Edition, Washington, DC: U.S. Government Printing Office, December 2010. Disponible en: http://www.naos.aesan.msssi.gob.es/naos/ ficheros/investigacion/Dietary_Guidelines_USA.pdf.

54. Salud MD.com. Nuevas recomendaciones sobre el consumo de azúcar. http://www.saludmd.com/noticias/ nuevas-recomendaciones-sobre-consumo-azucar.

55. Organización Mundial de la Salud (OMS) Informe sobre la situación mundial de las enfermedades no transmisibles 2010. Resumen de orientación. Ginebra, Suiza, 2011.

56. Organización Mundial de la Salud (OMS). Informe global sobre el alcohol y la salud. Ginebra, Suiza, 2011.

57. Organización Mundial de la Salud. Cancer. http://www. who.int/mediacentre/factsheets/fs297/es/index.html.

58. SINC, Servicio de Información y Noticias Científicas. La incidencia mundial de cáncer aumentará un 75\% en 2030 según la OMS. Disponible en: http://www.agenciasinc.es/Noticias/La-incidencia-mundial-de-canceraumentara-un-75-en-2030-segun-la-OMS. 